\title{
35 YEARS OF ADVANCEMENTS WITH THE COMPLEX VARIABLE BOUNDARY ELEMENT METHOD
}

\author{
NOAH J. DEMOES, GABRIEL T. BANN, BRYCE D. WILKINS, \\ THEODORE V. HROMADKA II, \& RANDY BOUCHER \\ Department of Mathematical Sciences, United States Military Academy, USA.
}

\begin{abstract}
The Complex Variable Boundary Element Method, or CVBEM, was first published in Journal of Numerical Methods in Engineering in year 1984 by authors Hromadka and Guymon [1]. Since that time, several papers and books have been published that present various aspects of the numerical technique as well as advances in the computational method such as extension to three or higher dimensions for arbitrary geometries, nonhomogeneous domains, extension to use of a Hilbert Space setting as well as collocation methods, inclusion of the time derivative via coupling to generalized Fourier series techniques, examination of various families of basis functions including complex monomials, the product of complex polynomials with complex logarithm functions (i.e., the usual CVBEM basis functions), Laurent series expansions, reciprocal of complex monomials, other complex variable analytic functions including exponential and others, as well as linear combinations of these families. Other topics studied and developed include rotation of complex logarithm branch-cuts for extension of the problem computational domain to the exterior of the problem geometry, depiction of computational error in achieving problem boundary conditions by means of the approximate boundary technique, mixed boundary value problems, flow net development and visualization, display of flow field trajectory vectors in two and three dimensions for use in depicting streamlines and flow paths, among other topics. The CVBEM approach has also been extended to solving partial differential equations such as Laplace's equation, Poisson's equation, unsteady flow equation, and the wave equation, among other formulations that include sources, sinks and combinations of these equations with mixed boundary conditions.

In the current paper, a detailed examination is made of the performance between four families of basis functions in order to assess computational efficiency in problem solving of two dimensional potential problems in a high aspect ratio geometric problem domain. Two selected problems are presented as case studies to demonstrate the different levels of success by each of the four families of examined basis functions. All four families involve basis functions that solve the governing partial differential equation, leaving only the goodness of fit in matching boundary conditions of the boundary value problem as the computational optimization goal. The modeling technique is implemented in computer programs Mathematica and MATLAB. Recommendations are made for future research directions and lessons learned from the current study effort.
\end{abstract}

Keywords: complex variables, complex variable boundary element method, CV BEM, basis functions, ideal fluid flow, Laplace's equation.

\section{INTRODUCTION}

The Complex Variable Boundary Element Method (CVBEM) has been the subject of numerous publications including papers and books [1-11]. The approximation approach solves several partial differential equations (PDE) of high interest to practitioners and developers in various aspects of engineering and computational mathematics. The modeling approach finds good use in potential flow problems, including heat, groundwater, diffusion and many other problems and applications of high interest in science, technology, engineering, and mathematics related topics. Most recently, the CVBEM has been extended into three or higher spatial dimensions for arbitrary geometric spatial domains [4], and has been applied to unsteady flow problems [8], and also problems involving the wave equation [12]. The modeling approach to applying the CVBEM to unsteady flow problems involves determination of the steady state condition and then reformulating the unsteady flow PDE into 
two portions involving the steady state portion and the unsteady flow portion. Under this scheme, the unsteady portion problem definition involves homogeneous boundary conditions, whereas the steady state portion problem definition involves the original problem boundary conditions. The unsteady portion is then solved by use of generalized Fourier series, and the steady state portion that includes the non-homogeneous boundary conditions, is solved by the CVBEM (or other Laplace's equation solver). The total solution is simply the sum of the solutions to the two problem portions identified in the above. The generalized Fourier series approach enables solution of a wide class of PDE given the homogeneous boundary conditions afforded by the CVBEM solution to the steady state portion of the problem. For example, a Poisson's equation problem with a constant load value of -2 is often used in the modeling of torsion problems. Similarly, the wave equation is solved by the Fourier series approach.

Since the introduction of the CVBEM in the Journal of Numerical Methods in Engineering [1], CVBEM technology has advanced over the last 35 years. The CVBEM technological advancements are briefly reviewed in this paper. The two-dimensional (2D) case is examined, although extension to high dimensions follows [4]. Then, focusing on the steady state portion of the PDE problem setting, the solution of the steady state portion of the PDE problem setting is examined in detail regarding the computational efficiency of different basis function families used in the CVBEM approach. The basis function families examined in the current paper are:

1. Complex Monomials, of the form $\left(z-z_{j}\right)^{n}$.

2. Products of complex linear monomials with complex natural logarithm functions (with rotated branch cuts), of the form, $\left(z-z_{j}\right) \ln \left(z-z_{j}\right)$.

3. Linear complex poles (inverse factors of the form, $\frac{1}{z-z_{j}}$, for several singularity points $z_{j}$, located exterior of the problem domain.

4. 4. Laurent series, of the form, $\sum_{j=1}^{n} \frac{1}{\left(z-z_{j}\right)^{j}}$.

Other complex analytic functions may be used as alternative basis function families, as can linear combination or other combinations of the basis functions that preserve the analytic definition of the overall CVBEM approximation function. Demonstration of basis function family computational efficiency is provided by examination of potential problems with respect to a high aspect ratio spatial domains located in the first quadrant of the complex plane.

The motivation for using complex analytic functions in the approximation approach includes the well-known properties of analytic functions. The analytic function involves two 2D real values functions that each solve Laplace's equation. Furthermore, these two real functions are conjugates which means that plots of isocontours of both functions result in the well-known flow net depiction of isopotentials and stream lines. These two real functions are called the real and imaginary components of the analytic function. And, these two components are related to each other by the Cauchy-Riemann equations, enabling both component functions to be examined given the other component function. Boundary conditions of the Dirichlet or flux type or even mixed types are all readily accommodated. Further details of the CVBEM are provided in the book, Foundations of the Complex Variable Boundary Element Method [4]. 
The CVBEM can be viewed as another generalized Fourier series that can be coupled to the usual Fourier series. In the current paper, the CVBEM approximation function is used to solve Laplace's equation (for example, the steady state portion of the transport equation) over the arbitrary geometric domain and with arbitrary but fixed boundary conditions. The usual Fourier series is used to solve the unsteady component of the governing PDE with homogeneous boundary conditions. Because of the use of the usual Fourier series, the geometric domains currently are limited to n-dimensional bricks in order for the Fourier series element functions to satisfy the homogeneous boundary conditions. Load terms involving sources and sinks are handled as part of the steady state portion of the PDE. A derivation and detailed application of the two-dimensional unsteady transport modeling approach based upon the CVBEM (where, as described previously, the CVBEM is used to solve the steady state portion of the unsteady flow problem, and Fourier series is used to solve the homogeneous unsteady portion of the transport problem) is demonstrated in the analysis of a groundwater mound unsteady flow problem $[13,14]$. Although the referenced application is focused upon an important computational geosciences problem, application of the CVBEM approach to other computational topics is straight forward [4].

\section{REVIEW OF THE CVBEM}

The CVBEM is an analytic method of solving boundary value problems that differs from conventional methods such as the finite difference method (FDM) and finite element method (FEM) that require interpolation to determine an approximation for the potential function. The CVBEM produces a continuous function that approximates the solution throughout the complete domain without the use of interpolation. The reason the CVBEM does not require interpolation is due to the method's reliance on analytic basis functions to form the solution approximation. Because the basis functions are analytic, the real and imaginary parts satisfy the Laplace equation. Because the basis functions are linear and homogeneous, the principle of superposition states the summation of the analytic functions also satisfies the Laplace equation that provides a stronger solution to the boundary value problem. The real part of the CVBEM approximation function represents the potential function while the imaginary part represents the streamlines. The real and imaginary parts of the approximation function are orthogonal to each other because the approximation function satisfies the Laplace equation.

The general CVBEM approximation function is

$$
\hat{\omega}(z)=\sum_{j=1}^{n} c_{j} g_{j}(z)
$$

where $\hat{w}(z)$ represents the CVBEM approximation for the potential at location $z, c_{j}$ represents the $j^{\text {th }}$ complex coefficient, and $g_{j}(z)$ represents the $j^{\text {th }}$ function in the set of complex basis functions chosen to approximate the global trial function, and $n$ represents the number of functions used to solve the global boundary value problem. In the following, the four basis function families considered in this paper will be examined. To solve the boundary value problem, the complex coefficients must be found. Each basis function that is used in the summation to approximate the potential function has a complex coefficient, comprised of both a real and imaginary part. Thus, there are 2 degrees of freedom for each basis function, and $2 n$ degrees of freedom for the approximate solution, where $n$ is the number of basis functions. To determine all coefficients there must be $2 n$ known potentials, $\omega(z)$, and their 
corresponding locations. To solve for the coefficients, create the following matrix equation of the form $\boldsymbol{G} \boldsymbol{c}=\boldsymbol{w}$ :

$$
\left[\begin{array}{lllll}
g_{00} & g_{01} & g_{02} & \cdots & g_{02 n} \\
g_{10} & g_{11} & g_{12} & \cdots & g_{12 n} \\
g_{20} & g_{21} & g_{22} & \cdots & g_{22 n}
\end{array}\right]\left[\begin{array}{l}
c_{0} \\
c_{1} \\
c_{2} \\
\vdots \\
c_{2 n}
\end{array}\right]=\left[\begin{array}{l}
w_{0} \\
w_{1} \\
w_{2} \\
\vdots \\
w_{2 n}
\end{array}\right]
$$

where $\boldsymbol{G}$ represents the value of each basis function at $z_{j}, \boldsymbol{c}$ represents the vector of complex coefficients, and $\boldsymbol{w}$ represents the known potential values. Solve for the coefficients by performing linear algebra operations to row reduce the matrix to find $c$. Once the coefficients are found and back substituted into the CVBEM approximation function, the real part is used to approximate the potentials in the problem domain.

In this paper, basis function families are used without combination. However, another option for use in the approximation approach is to use a linear combination of the various basis function families. Additionally, other analytic functions are available to use as basis functions such as the complex exponential function and complex trigonometric functions (that are, in turn, related to the complex exponential functions). Research into the CVBEM appears to indicate that use of the basis functions developed by the numerical integration of the Cauchy integral formula results in the most stable and usable basis functions.

\section{CVBEM BASIS FUNCTION FAMILIES CONSIDERED}

The general CVBEM function is

$$
\hat{w}(z)=\sum_{j=1}^{n} c_{j} g_{j}(z)
$$

\subsection{Complex Monomials}

For this basis function family, complex functions of the form $\left(z-z_{j}\right)^{n}$ are used where coordinates $z_{j}$ are expansion points as utilized in the Taylor series for complex variable functions [15]. Expansion of these basis functions reduces to the simpler complex monomials of the form $z^{n}$, where as in the prior form, $n$ are positive integer exponents. This formulation is another representation of the complex variable polynomial method or CPM, as examined in a number of publications [16]. The CPM approximation function is

$$
\hat{w}(z)=\sum_{j=1}^{n} c_{j}\left(z-z_{j}\right)^{n}
$$

3.2 Numerical Integration of Cauchy Integral Using Polynomial Interpolation Functions (Standard CVBEM Basis Functions)

The more traditional basis function family is of the form $\left(z-z_{j}\right) \ln { }_{j}\left(z-z_{j}\right)$ where points $z_{j}$ are CVBEM nodes and the $\ln _{j}\left(z-z_{j}\right)$ is the complex natural logarithm function defined 
with its branch cut selected to lie exterior of the problem domain union boundary. Although nodal coordinates $z_{j}$ typically are defined on the problem boundary [4], placing CVBEM nodes to be located exterior of the problem domain union boundary have also been used with good computational results [17]. These basis functions result from the numerical integration of the Cauchy integral formula where linear trial functions are used to interpolate the complex function between nodes located on the problem boundary. Depending on mathematical formulation, different numerical statements can result that all have in common the basis functions as described above. The standard CVBEM approximation function is

$$
\hat{w}(z)=\sum_{j=1}^{n} c_{j}\left(z-z_{j}\right) \ln _{j}\left(z-z_{j}\right)
$$

\subsection{Complex Poles}

Another basis family family examined are the traditional complex variable poles of the form $\frac{1}{z-z_{j}}$. The points $z_{j}$ are located on or the problem boundary or exterior of the problem domain union boundary. Theoretical considerations involving complex function poles of higher orders are examined in standard texts on complex variables [15]. The complex poles CVBEM approximation function is

$$
\hat{w}(z)=\sum_{j=1}^{n} c_{j} \frac{1}{z-z_{j}}
$$

\subsection{Laurent Series}

The fourth family of basis functions examined is the terms of the Laurent series and are of the form $\frac{1}{\left(z-z_{0}\right)^{n}}$, where $n$ are positive integers and $z_{0}$ is a fixed expansion location exterior to the problem domain and usually also exterior to the problem boundary. The location of $z-0$ impacts the approximation error. In the following, $z_{0}=40+40 i$ because this value reduced the approximation error significantly compared to other locations such as $-40-40 i$ or $20+$ 20i. Several Laurent series can be examined together, melding together some of the advantages of the complex function poles basis functions. However, $z_{0}$ must be located outside the problem domain to enable the basis function to be completely analytic on the problem domain. The theoretical exploration of the Laurent series can be found in other books [15]. The Laurent series CVBEM approximation function is

$$
\hat{w}(z)=\sum_{j=1}^{n} c_{j} \frac{1}{\left(z-z_{0}\right)^{n}}
$$

3.5 Mixed Basis Function Approximations and other Families of Basis Functions

Another option for use in the approximation approach is to use a linear combination of the various basis functions examined in the above. Additionally, other analytic functions are 
available to use as basis functions such as the complex exponential function and complex trigonometric functions (that are, in turn, related to the complex exponential functions). Research into the CVBEM appears to indicate that use of the basis functions developed by the numerical integration of the Cauchy integral formula results in the most stable and usable basis functions.

\subsection{Example Problems}

In order to demonstrate the utility and efficiency of the CVBEM and the various basis functions considered in the above, two test problems are examined:

1. Heat transfer in a two dimensional rod.

2. Ideal fluid flow in a 90-degree bend.

Both demonstrations are considered in detail in Section 4. To determine the accuracy of the CVBEM using different basis functions, the exact solution is compared to the CVBEM approximation function along the center line of the domain. The reason the center line is chosen is twofold. The first reason is because the center line encompasses the largest deviation to the exact solution due to the decrease in proximity to the boundary. The second reason is because the center line is the most accurate depiction of the interior which is the part of the domain that the CVBEM is designed to model. The first problem tests the computational accuracy of the CVBEM against the FEM in high aspect ratio domains with simple boundary conditions. The second example problem tests the effectiveness of different basis functions as the boundary condition complexity increases in the high aspect ratio domain to determine if certain basis functions are more accurate for high aspect ratio domains. The last two example problems examine other features of the CVBEM, such as the inclusion of the time derivative as used in transient potential flow problems or the wave equation, among other formulations, as well as sources and sinks, and considerations of three or higher spatial dimensions, are presented in the referenced publications above.

\section{EXAMPLE PROBLEM DOMAIN DEFINITION}

The problem domain is a two-dimensional spatial rectangle of high aspect ratio. The standard domain has top and bottom boundaries of length 10 and the left and right boundaries of length 1 . All example problem places the problem domain entirely in the first quadrant. The standard problem domain is depicted in Fig. 1 where the boundary conditions are represented as $u_{n}$, for the $n^{\text {th }}$ test problem.

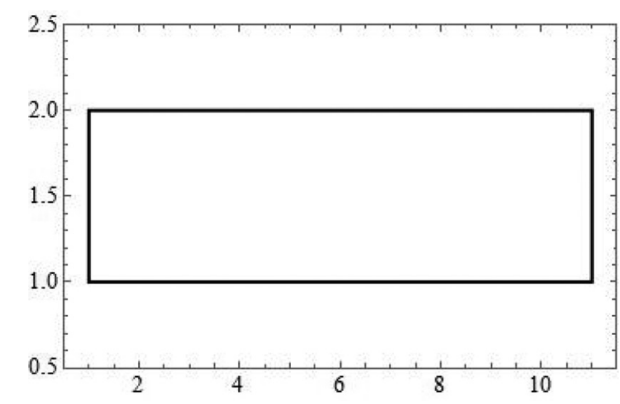

Figure 1: Standard problem domain for both test problem. 


\section{TEST PROBLEM 1: HEAT TRANSFER IN A ONE DIMENSIONAL ROD}

\subsection{Description of Test Problem 1 - Numerical Solutions and Assessments}

Heat transfer is one of the many applications that Laplace's equation can model. Laplace's equation is of the form

$$
\frac{\partial u_{1}}{\partial x^{2}}+\frac{\partial u_{1}}{\partial y^{2}}=0
$$

and is used in combination with the boundary conditions to solve for $u_{1}(x, t)$, the approximate solution to Test Problem 1. In this one dimensional rod, heat travels linearly through the rod on the top and bottom edges of the rod for this problem while the temperatures on the left and right boundary conditions are held at constant temperatures, 0 and 1, respectively. The boundary conditions on the top and bottom must equal the boundary conditions on the left and right at each corner of the rod. The global boundary value problem boundary conditions satisfy these conditions are:

$$
\begin{aligned}
& u_{1}(1, y)=0, \quad u_{1}(x, 1)=\frac{x}{10}-\frac{1}{10} \\
& u_{1}(11, y)=1, \quad u_{1}(x, 2)=\frac{x}{10}-\frac{1}{10}
\end{aligned}
$$

Because the length is known and the boundary conditions are a function of location and length all temperatures are known along the boundary. To solve for the temperatures on the inside of the rod, collocation points are chosen at evenly spaced locations along the boundaries and the known boundary temperatures are used to solve for the complex coefficients in the approximation functions. The following sections provide the results of the approximation functions compared to the exact solution along the center line of the domain. The center line is used for accuracy analysis within the domain because that line is furthest from either boundary, making the center line the line in which approximation error will be the greatest within the boundary domain. Examination of the domain where the maximum error occurs provides clarity on the accuracy of the approximation within the entire domain because all other approximation error within the domain is less. The following sections display the results.

\subsection{Comparison of CVBEM Basis Functions with Linear Boundary Conditions}

The CPM, CVBEM and complex poles basis function approximation function use $n=32$ collocation points to solve for the approximation functions. The Laurent series uses $n=17$ instead of $n=32$ because $n=17$ provides a more approximate solution. Figure 2 and 3 break up the four approximation functions into two groups of two plotted against the exact solution. In Fig. 2, the exact solution of the problem which is known because test problem 1's boundary conditions are continuous throughout the problem domain is plotted against the CPM and CVBEM approximation method. There appears to be no significant error between the CPM and CVBEM approximations and the exact solution. The same conclusion is made about the Laurent series approximation and the complex poles basis function approximation in Fig. 3. 


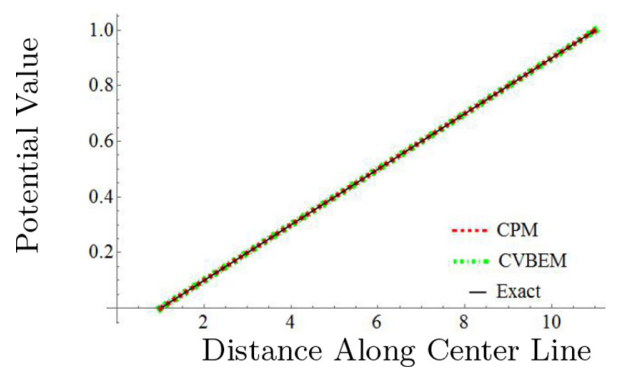

Figure 2: CPM and CVBEM vs exact.

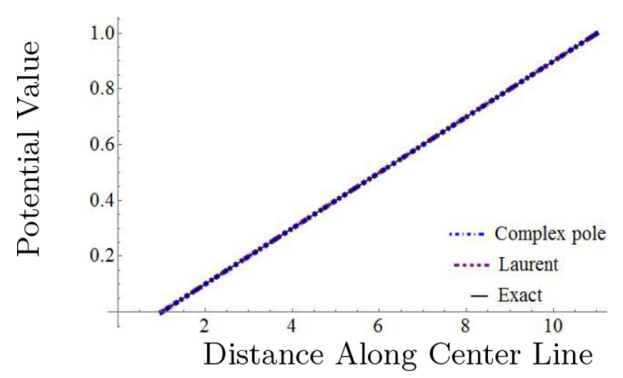

Figure 3: Laurent and complex poles vs exact.

Computational error analysis is depicted in Figs. 4-5. For this paper computation error is defined as

$$
C E=\frac{\left|f(x, y)-u_{n}(x, y)\right|}{|f(x, y)|}
$$

where CE is computational error, $f(x, y)$ is the exact solution, and $u_{n}(x, y)$ is the $n$ type of basis function approximation. Figure 4 shows the amount of computational error for the entire length of the domain on the center line for the CPM and CVBEM approximation. Figure 5 provides the same information for the Laurent and complex poles basis function approximations.

The CPM, CVBEM, and complex poles basis function approximation all produce better approximations for the boundary value problem than the Laurent series approximation. Each

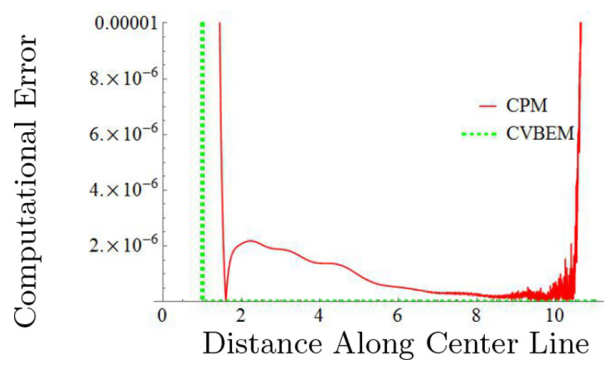

Figure 4: CPM and CVBEM error 


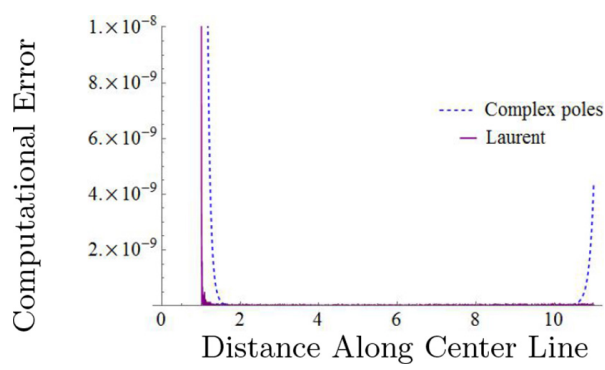

Figure 5: Laurent and complex poles Error.

type of basis function acts differently. Thus, some basis functions are more suitable for specific types of modeling problems than other basis functions.

Additionally, the figures above depict the error along the center line. This error distribution is in line with the Min/Max Modulus Theorem. Because the max error occurs along the boundary, examination of the error along the boundary is only necessary, reducing computational cost.

\section{TEST PROBLEM 2: FLUID FLOW IN A 90-DEGREE BEND}

Ideal fluid flow in a 90-Degree Bend can be modeled by the function $f(z)=z^{2}$. The real part of $f(z), x^{2}-y^{2}$ represents the isopotential values. Because fluid flow potentials are dependent on location, the boundary conditions on all four sides of the domain are of the same form, $x^{2}$ $-y^{2}$. The domain in this problem is initially set to a 1:10 ratio. Section 6.2, depicts the accuracy of the four basis function approximations to the exact solution along the center line. The same boundary conditions are applied to two other high aspect ratio domains or ratios 1:25 and 1:50 to determine the maximum absolute error for each approximation compared to the error of the FEM discrete approximation.

\subsection{Description of Test Problem 2 - Numerical Solutions and Assessments}

Similar to Test Problem 1, Laplace's equation

$$
\frac{\partial u_{2}}{\partial x^{2}}+\frac{\partial u_{2}}{\partial y^{2}}=0
$$

is the governing PDE that must be solved to create an approximation for test problem 2 . The global boundary value problem boundary conditions are given by:

$$
\begin{array}{ll}
u_{2}(1, y)=x^{2}-y^{2}, & u_{2}(11, y)=x^{2}-y^{2}, \\
u_{2}(x, 1)=x^{2}-y^{2}, & u_{2}(x, 2)=x^{2}-y^{2} .
\end{array}
$$

\subsection{Comparison of CVBEM Basis Function Performance}

Figure 6 shows the comparison of the two most accurate basis function approximations plotted against the exact solution, $x^{2}-y^{2}$. Ocular analysis determines no significant difference between the exact solution and the approximation solutions for the CPM and CVBEM basis functions. Similarly Fig. 7 depicts the Laurent series and Complex Poles approximation 


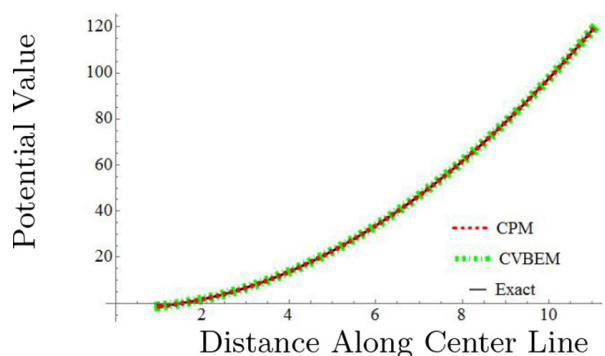

Figure 6: CPM and CVBEM vs exact.

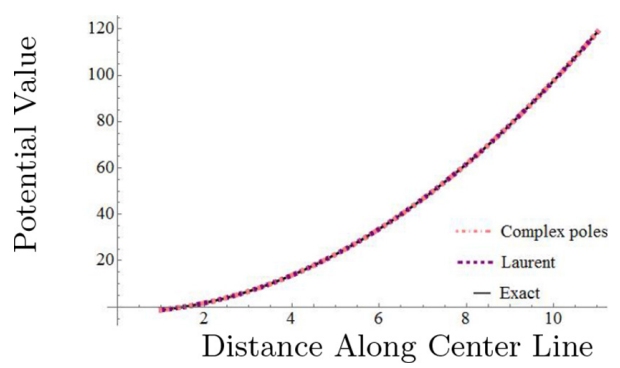

Figure 7: Laurent and complex poles vs exact.

function with $n=17$ collocation points and $n=32$ collocation points, respectively. The reason that the Laurent series used 17 collocation points instead of $n=32$ like the other three basis functions is do to the increased error when $n$ increases for the Laurent Series. This is due to the computers ability to handle the ill-conditioned matrix caused by the nature of the basis function. Figures 8 and 9 depict the error, defined to be the difference between the exact solution and the approximate solution on the center line, for each approximation function. The center line is defined to be the the line bisecting the problem domain at $y=1.5$.

The error is significantly less for the CPM than for any other basis function approximation. The reason for this is that the problem that is being modeled $u_{2}(x, y)=x^{2}-y^{2}$ is a part of the basis function family that makes up the CPM approximation. Thus, the exact solution is contained in the CPM approximation, resulting in zero error. This would not occur in an application problem, but is important to understand.

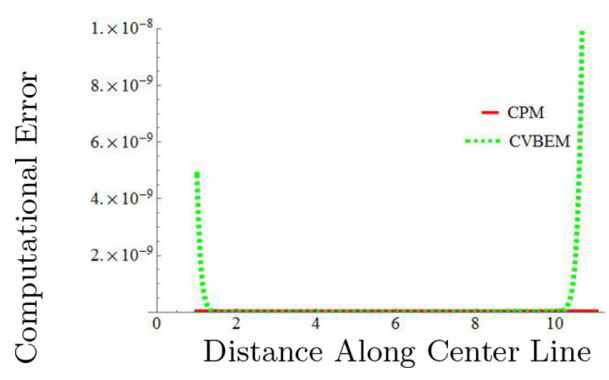

Figure 8: CPM and CVBEM error. 


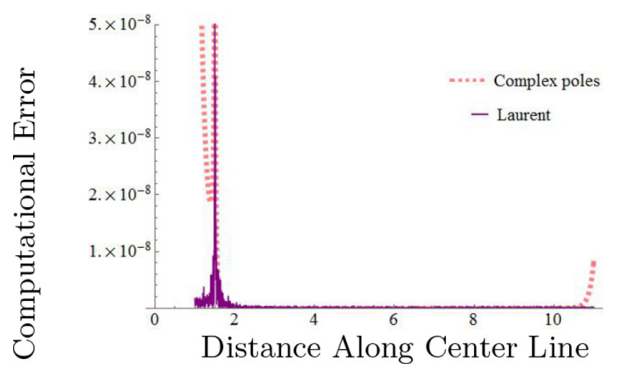

Figure 9: Laurent and complex poles error.

\subsection{Error Comparison for Different Domain Ratio}

Additional analysis on the impact domain ratio has on the accuracy of the CVBEM resulted in testing three different domain ratios across the four basis function families used in the CVBEM, complex monomials, traditional CVBEM, complex poles, and Laurent series using $n=128$ collocation points. The maximum computational error of each approximation is compared to the computational error of the FEM that uses a step size of 0.1. The error analysis is conducted on Test Problem 2. The results are listed in Table 1.

The error data reveals that the higher the domain ratio, the greater the error for each. While the CPM and complex poles basis function family error decrease significantly, the CVBEM appears to maintain its validity with limited change in error. Additionally, the Laurent series basis family produces the most inaccurate model. However, the FEM is the most accurate method of modeling BVPs in high aspect ratio domains out of the basis function families tested.

There are two reasons for why the FEM produced a better approximation for this problem. The computer and software used to create our approximation function fails to accurately solve for the ill-conditioned matrix that is created when solving for the coefficients. As a result, the complex approximation functions are limited to $n=128$ basis functions. If the program and computational ability increased and the number of basis functions increased there would be higher accuracy in the complex approximation functions that may be greater than the FEM. The second effect of having $n$ limited to 128 is that there is a greater gap between each of the collocation points along the problem domain. This results in greater ambiguity in the approximation function as the space between known potential values increases with a greater aspect ratio.

Although these conclusions are true, node count is not totally indicative of modeling performance because the positioning of the model nodes and collocation points can have

Table 1: Display of error for different basis families.

Maximum Relative Error on the Boundary $n=128$

\begin{tabular}{llllll}
\hline Domain Ratio & CPM & CVBEM & Complex poles Laurent & FEM \\
\hline $1: 10$ & $1.023 \times 10^{-12}$ & $6.821 \times 10^{-13}$ & $1.463 \times 10^{-12}$ & $3.412 \times 10^{-09}$ & $3.979 \times 10^{-13}$ \\
$1: 25$ & $1.743 \times 10^{-10}$ & $2.046 \times 10^{-12}$ & $3.798 \times 10^{-10}$ & $1.942 \times 10^{-07}$ & $1.932 \times 10^{-12}$ \\
$1: 50$ & $3.919 \times 10^{-09}$ & $2.971 \times 10^{-10}$ & $9.780 \times 10^{-10}$ & $2.512 \times 10^{-05}$ & $6.366 \times 10^{-02}$ \\
\hline
\end{tabular}


considerable influence on computational results. Current research shows that similar computational error may be achieved with fewer nodes and collocation points by careful positioning of the nodes and collocation points.

\section{CONCLUSIONS AND RECOMMENDATIONS FOR FUTURE RESEARCH}

The Complex Variable Boundary Element Method has the capability to use different basis functions as the analytic functions to approximate the potential function. In this paper, error for each basis function was examined as the domain ratio decreases. The reason for the increase in error occurs because of the limitations in computing power. The computers used in these tests were only able to use up to $n=128$ collocation points. Thus, as the domain stretches with higher aspect ratios, the distance between each collocation point increases, increasing the probability that the approximate solution estimates an incorrect potential value. Although error increased for each basis function family that was tested, the traditional CVBEM model with basis functions of the form $\left(z-z_{j}\right) \ln { }_{j}\left(z-z_{j}\right)$ decreased in accuracy the slowest as the domain ratio increased; therefore, the traditional CVBEM holds its accuracy the best in problems with high aspect ratios.

In Table 1, the CVBEM demonstrated that the error decreased between the ratios of 1:1 and 1:10 before increasing for higher domain ratios. Future research includes determining the domain ratio that optimizes accuracy for each basis function and the specific boundary conditions. Once the basis function is paired with the domain ratio that optimizes CVBEM performance, the research can be extended to applying the basis function family to 3 dimensional domains with the same high aspect ratio.

\section{REFERENCES}

[1] Hromadka, T.V. \& Guymon, G.L., Numerical analysis of normal stress in nonNewtonian boundary layer flow. Journal of Numerical Methods in Engineering, 20(1), pp. 25-37, 1984.

[2] Hromadka, T.V. \& Lai, C., The Complex Variable Boundary Element Method. SpringerVerlag: New York, 1984.

[3] Hromadka, T.V. \& Whitley, R.J., Approximating three-dimensional steady state potential flow problems using two-dimensional complex polynomials. Engineering Analysis with Boundary Elements, 29, pp. 190-194, 2005. https://doi.org/10.1016/j.enganabound.2004.07.004

[4] Hromadka, T.V. \& Whitley, R.J., Foundations of the Complex Variable Boundary Element Method. Springer: New York, 2014.

[5] Hromadka, T.V. \& Whitley, R.J., A new formulation for developing CVBEM approximation functions. Engineering Analysis with Boundary Elements, 29(1), 39-41, 1996. https://doi.org/10.1016/s0955-7997(96)00027-6

[6] Dumar, P. \& Kumar, R., Complex variable boundary element method for torsion in anisotropic bars. Applied Mathematical Modeling, 17(2), pp. 80-88, 1993. https://doi.org/10.1016/0307-904x(93)90096-y

[7] Hromadka, T.V. \& Whitley, R., Advances in the Complex Variable Boundary Element Method. Springer-Verlag: New York, 1998. 
[8] Wilkins, B.D., Greenberg, J., Redmond, B., Baily, A., Flowerday, N., Kratch, A., Hromadka, T.V., Boucher, R., McInvale, H.D. \& Horton, S., An unsteady two dimensional complex variable boundary element method. Applied Mathematics, 8(6), pp. 878-891, 2017.

https://doi.org/10.4236/am.2017.86069

[9] Hromadka, T.V., A Multi-Dimensional Complex Variable Boundary Element Method. WIT Press: Southampton, England, 2002.

[10] Hromadka, T.V. \& Lai, C., The Complex Variable Boundary Element Method in Engineering Analysis. Springer-Verlag: New York, First print 1987, Republished Digitally, 2012.

[11] Hromadka, T.V., Isenhour, M., Rao, P., Yen, C.C. \& Crow, M., Computational biopsy to assess accuracy of large scale computational groundwater flow models. International Journal of Engineering Sciences and Management, 7(1), pp. 6-11, 2017.

[12] Wilkins, B.D., Hromadka, T.V. \& Boucher, R., A conceptual numerical model of the wave equation using the complex variable boundary element method. Scientific Research Publishing, 8(5), pp. 724-735, 2017.

https://doi.org/10.4236/am.2017.85057

[13] Wilkins, B.D., Flowerday, N., Kratch, A., Greenberg, J., Redmond, B., Hromadka, T.V., Hood, K., Boucher, R. \& McInvale, H.D., A computational model of groundwater mound evolution using the complex variable boundary element method and generalized fourier series. The Professional Geologist, 54(1), pp. 12-15, 2017.

[14] Hood, K., Wilkins, B.D., Hromadka, T.V., Boucher, R. \& McInvale, H.D., Development of an earthen dam break date base. The Professional Geologist, 54(1), 10-11, 2017.

[15] Matthews, J.H. \& Howell, R.W., Complex Analysis for Mathematics and Engineering. Times Mirror Company: Iowa, 1996.

[16] Bohannon, A.W. \& Hromadka, T.V., The complex polynomial method with a leastsquares fit to boundary conditions. Engineering Analysis with Boundary Elements, 33(8-9), pp. 1100-1102, 2009. https://doi.org/10.1016/j.enganabound.2009.02.005

[17] Bloor, C., Hromadka, T.V., Wilkins, B.D. \& McInvale, H.D., Cvbem and fvm computational model comparison for solving ideal fluid flow in a 90-degree bend. Open Journal of Fluid Dynamics, 6, pp. 430-437, 2016. https://doi.org/10.4236/ojfd.2016.64031 